\title{
Do human screams permit individual recognition?
}

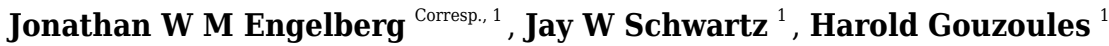 \\ 1 Department of Psychology, Emory University, Atlanta, Georgia, United States \\ Corresponding Author: Jonathan W M Engelberg \\ Email address: jonathan.engelberg@emory.edu
}

The recognition of individuals through vocalizations is a highly adaptive ability in the social behavior of many species, including humans. However, the extent to which nonlinguistic vocalizations such as screams permit individual recognition in humans remains unclear. Using a same-different vocalizer discrimination task, we investigated participants' ability to correctly identify whether pairs of screams were produced by the same person or two different people, a critical prerequisite to individual recognition. Despite prior theory-based contentions that screams are not acoustically well-suited to conveying identity cues, listeners discriminated individuals at above-chance levels by their screams, including both acoustically modified and unmodified exemplars. We found that vocalizer gender explained some variation in participants' discrimination abilities and response times, but participant attributes (gender, experience, empathy) did not. Our findings are consistent with abundant evidence from nonhuman primates, suggesting that both human and nonhuman screams convey cues to caller identity, thus supporting the thesis of evolutionary continuity in at least some aspects of scream function across primate species. 
8

9 DO HUMAN SCREAMS PERMIT INDIVIDUAL 10 RECOGNITION?

Jonathan W. M. Engelberg ${ }^{1}$, Jay W. Schwartz ${ }^{1}$, Harold Gouzoules ${ }^{1}$

${ }^{1}$ Department of Psychology, Emory University, Atlanta, GA, United States

Corresponding Author:

17 Jonathan Engelberg,

Department of Psychology, Emory University, 36 Eagle Row, Atlanta, GA, 30322, United

States.

E-mail: jonathan.engelberg@emory.edu. 


\section{ABSTRACT}

26 The recognition of individuals through vocalizations is a highly adaptive ability in the social

27 behavior of many species, including humans. However, the extent to which nonlinguistic

28 vocalizations such as screams permit individual recognition in humans remains unclear. Using a

29 same-different vocalizer discrimination task, we investigated participants' ability to correctly

30 identify whether pairs of screams were produced by the same person or two different people, a

31 critical prerequisite to individual recognition. Despite prior theory-based contentions that

32 screams are not acoustically well-suited to conveying identity cues, listeners discriminated

33 individuals at above-chance levels by their screams, including both acoustically modified and

34 unmodified exemplars. We found that vocalizer gender explained some variation in participants'

35 discrimination abilities and response times, but participant attributes (gender, experience,

36 empathy) did not. Our findings are consistent with abundant evidence from nonhuman primates,

37 suggesting that both human and nonhuman screams convey cues to caller identity, thus

38 supporting the thesis of evolutionary continuity in at least some aspects of scream function

39 across primate species. 


\section{INTRODUCTION}

49

50

51

52

For many species, the ability to recognize individuals by distinctive cues or signals is essential to the organization of social behavior (Seyfarth \& Cheney, 2015; Steiger \& Müller, 2008; Tibbetts \& Dale, 2007; Yorzinski, 2017). Research spanning a variety of taxa, contexts, and modalities has suggested that recognition is broadly functional, facilitating behaviors directed at specific individuals (or classes thereof, such as kin) and possibly enabling opportunities to learn about third-party social dynamics (Seyfarth \& Cheney, 2015). Recognition from vocalizations, in particular, has received attention in primates and other taxonomic groups for its potential to benefit listeners who might lack other sources of information, e.g., if the vocalizer is distant or visually occluded (Charrier, Pitcher, \& Harcourt, 2009; Seyfarth \& Cheney, 2015).

For speech or speech-derived signals, humans are adept at making identity-related judgments on the basis of vocal cues alone (Perrachione, Del Tufo, \& Gabrieli, 2011; Remez et al., 1997; Sheffert, Pisoni, Fellowes, \& Remez, 2002; reviewed in Mathias \& von Kriegstein, 2014). Listeners can infer information relating to a speaker's identity even when a vocal signal has been heavily altered or reduced (Gonzalez \& Oliver, 2005; Remez, Fellowes, \& Rubin, 1997; Van Lancker, Kreiman, \& Emmorey, 1985). However, less is known about the extent to which humans perceive and respond to identity cues in natural, nonlinguistic vocalizations such as laughter and screams. In many ways, these vocalizations seem less similar to speech than they are to the calls of nonhuman species, in terms of their acoustic structures (Bryant \& Aktipis, 2014; Davila-Ross, Owren, \& Zimmerman, 2009; Lingle, Wyman, Kotrba, Teichroeb, \& Romanow, 2012; McCune, Vihman, Roug-Hellichius, Delery, \& Gogate, 1996), the neural mechanisms associated with them (Belin, 2006; Owren, Amoss, \& Rendall, 2011), and perhaps 
71 in some of their social communicative functions as well (McCune et al., 1996; Owren et al.,

72 2011). Research in this understudied area is therefore significant with respect to understanding

73 the functions and evolution of the human vocal repertoire.

74 Thus far, research on infant cries has demonstrated that mothers and fathers can

75 discriminate their own child's vocalizations from those of unrelated individuals (Green \&

76 Gustafson, 1983; Gustafson, Levréro, Reby, \& Mathevon, 2013), a finding consistent with

77 evidence from a variety of non-human taxa showing that parents recognize their own offsprings'

78 calls (Beecher, 1990; Charrier et al., 2009; Knörnschild, Feifel, \& Kalko, 2013). More recent

79 research has shown that humans can discriminate identity from laughter (although the ability to

80 do so is impaired in spontaneous, relative to volitional, laughter; Lavan, Scott, \& McGettigan,

81 2016; Lavan, Short Wilding, \& McGettigan, 2018), and can make accurate, identity-related

82 judgments from human "roars" (Raine, Pisanski, Oleszkiewicz, Simner, \& Reby, 2018). Whether

83 human screams permit similar discrimination remains unexplored, but some comparative

84 evidence suggests that the acoustic structures of these vocalizations might enable identity-related

85 judgments. Similarly loud, high-pitched distress calls in species of birds (Rohwer et al., 1976)

86 and mammals (Lingle, Rendall, \& Pellis, 2007) elicit altruistic responses from kin, implying that

87 they may possess features sufficient to distinguish relatedness, if not more precise individually

88 distinctive attributes.

89 Many species of non-human primates produce screams primarily during agonistic

90 conflicts (Cheney, 1977; de Waal \& van Hooff, 1981). Research from our lab has documented

91 the role of these screams in evoking aid from kin or other allies (Gouzoules, Gouzoules, \&

92 Marler, 1984; Gouzoules, Gouzoules, \& Marler, 1986), with significant implications for

93 agonistic outcomes and the acquisition and maintenance of rank in the dominance hierarchy 
94 (Cheney \& Seyfarth, 1990; Silk, 2002; Gouzoules, 2005). Importantly, these vocalizations

95

96

97

98

transmit over long distances (Gouzoules \& Gouzoules, 2000), and so are often heard by listeners lacking other situational information concerning the caller. Therefore, one might expect that screams should convey cues to kinship or identity. Evidence from numerous studies suggest that primates recognize individuals from their screams (Bergman, Beehner, Cheney, \& Seyfarth, 2003; Cheney \& Seyfarth, 1980; Fugate, Gouzoules, \& Nygaard, 2008; Gouzoules et al., 1986; Kojima, Izumi, \& Ceugniet, 2003; Seyfarth \& Cheney, 2015; Slocombe, Kaller, Call, \& Zuberbühler, 2010). Indeed, a number of playback studies investigating other cognitive abilities such as hierarchical classification (Bergman et al., 2003) and third-party social inference (Slocombe et al., 2010) have taken as a basic assumption that listeners can recognize the identity of a screamer.

Despite this behavioral evidence, some researchers have argued that screams are not acoustically well-suited for communicating identity-related cues. Most notably, Rendall and colleagues contended that primate screams lack the individual distinctiveness of other calls, as they are too high in fundamental frequency (yielding sparser harmonic structures) and too chaotic (lacking a well-defined periodic structure) to reveal consistent, individual-specific markers of supralaryngeal filtering (Owren \& Rendall, 2003; Rendall, Owren, \& Rodman, 1998; Rendall, Notman, \& Owren, 2009). These researchers found that rhesus macaque (Macaca mulatta) and human listeners alike were better able to discriminate individual macaques on the basis of their 'coos' - stable, harmonically rich vocalizations - than by their screams, although, notably, humans still discriminated above chance when listening to screams (Owren \& Rendall, 2003). A follow-up study by Fugate et al. (2008) also showed that listeners of both species were capable of discriminating rhesus macaques by their screams, but findings underscored the 
117 importance of taking into account factors that may underlie variation in response (e.g.,

118 dominance rank in macaques).

More recently, Hansen, Nandwana, and Shokouhi (2017) acoustically analyzed human

120 screams in an effort to develop automated systems that recognize speakers on the basis of their

121 screams. They reported that screams exhibited substantial acoustic variability both between and

122 within vocalizers, consistent with Owren and Rendall's (2003) contention that screams are,

123 overall, unpredictable and perhaps unsuited for conveying identity. Indeed, Hansen et al. (ibid.)

124 found that human listeners, when presented two successive screams, were unable to identify

125 whether or not those screams were produced by the same vocalizer. However, this perceptual test

126 was clearly not the primary focus of their study: only a very small number of participants $(\mathrm{N}=$

127 10) completed a relatively small number of trials (10 involving two screams, whereas 10

128 required matching screams to speech). Thus, we suggest that it remains an open question as to

129 whether human listeners can perceive anything about identity from screams. A more in-depth

130 examination of human perceptual abilities in this area is needed, especially given the rich,

131 comparative data suggesting that acoustically analogous vocalizations do permit some judgments

132 about caller identity in other species (Gouzoules et al., 1986; Kojima et al., 2003).

In the present study, participants were tested in a same-different identity discrimination

task, similar to the listening tasks used in prior studies (Fugate et al., 2008; Hansen et al., 2017;

135 Owren \& Rendall, 2003), wherein they decided whether two, sequentially presented screams

136 were produced by the same vocalizer or by two different vocalizers. This goal of this task was

137 not to test individual recognition per se-listeners were not identifying specific individuals by

138 their screams - but instead to test the critical prerequisite that human screams, like those of

139 nonhuman primates, furnish perceptible cues to a vocalizer's identity. We hypothesized that if 
140 human screams convey identity cues, then listeners would perform above chance at

141 discriminating vocalizers on the basis of screams, even if screams may lack some of the reliable,

142 self-identifying attributes of speech or other, more periodic and/or low-pitched vocalizations.

143 An additional aim was to identify potential sources of variability in accuracy and

144 response latency on the task. First, we assessed the role of both screamer and listener gender on

145 patterns of individual recognition. Gender seems to explain some variation in identification of

146 emotions from non-linguistic vocalizations, with female listeners showing slightly higher

147 accuracy than males, and vocalizer gender having mixed effects on listener accuracy (Lausen \&

148 Schacht, 2018). However, few studies have explored the effects of sex or gender on the auditory

149 perception of identity in nonlinguistic vocalizations, with the exception of research on infant

150 cries, which has focused on potential differences between mothers and fathers and has yielded

151 mixed results (Gustafson et al., 2013). In addition to gender-related factors, we investigated a

152 possible effect of exposure to screams through the media (as assessed by a questionnaire

153 developed in our lab); it has been suggested that experience with a vocal class may facilitate the

154 recognition of identity from vocalizations of that kind (Lavan, Burton, Scott, \& McGettigan,

155 2018). We also examined the effect of empathy (as measured by the Cambridge Behaviour

156 Scale; Baron-Cohen \& Wheelwright, 2004), which has been shown to underlie variation in

157 response to other distress vocalizations (Wiesenfeld, Whitman, \& Malatesta, 1984) and is

158 particularly relevant in underpinning responses to screams (de Waal, 2008).

\section{MATERIALS \& METHODS}

160 Testing took place over a two-year period at Emory University's Bioacoustics Laboratory

161 in the Department of Psychology. This research was conducted in compliance with Emory's

162 Institutional Review Board under IRB00051516, approved July 26, 2011. 


\section{Participants}

164

165

166

167

168

169

170

171

172

173

174

175

176

177

178

179

180

181

182

183

184

The participant pool consisted of 104 volunteers from Emory University, including 73

females and 31 males. Ages ranged from 17 to 41 years $(M=19.53, S D=2.48)$. The majority of participants were undergraduates, recruited via an online portal system, who received class credit for completion of the study. All participants provided their voluntary and informed written consent.

\section{Materials}

Stimuli. Scream exemplars were compiled from commercial movies, scripted and unscripted television programs, newscasts, advertisements, online sources such as YouTube, and commercially available sound banks (Human Sound Effects, Partners In Rhyme, Inc., Santa Monica, CA; The Nightingale Voice Box, Nightingale Music Productions, North York, Ontario, Canada). Screams comprise a salient and readily identified natural category within the human nonlinguistic vocal repertoire (Anikin, Bååth, \& Persson, 2018). Accordingly, we were able to identify screams for use in this study by ear, without utilizing formal acoustical selection criteria (which, in any event, are not fully described in the literature). In another study (Schwartz, Engelberg, \& Gouzoules, under review), participants classified as screams a large subset of the exemplars used here; all of the exemplars in that subset were classified as screams at rates of $>$ $90 \%$. Screams were selected on the basis of sound quality (i.e., minimal noise; no overlapping sounds), and to represent a variety of emotional contexts (e.g., fear, excitement, anger, pain).

Online videos were downloaded using Total Recorder version 8.0 (High Criteria, Inc., Richmond Hill, Ontario, Canada) and WinXHD Video Converter Deluxe (Digiarty Software, Inc., Chengdu, China), while DVD media were extracted using WinXDVD Ripper Platinum (Digiarty 
185 Software, Inc., Chengdu, China). All source videos were saved, converted to the MPEG file 186 format, and cropped at timestamps surrounding the target vocalizations.

Audition CC (Adobe Systems, San Jose, CA) and Audacity version 2.1.2

189

190

191

192

193

194

195

196

197

198

199

200

201

202

203

204

205

206

207

(http://audacity.sourceforge.net). Edits were applied when necessary to delete clicks and pops

(Owren \& Bachorowski, 2007) or mitigate noise without distorting or interfering with the acoustics of the screams themselves (as determined by listening and by visual inspection of spectrograms). Additionally, in the case of some DVD sources, separate tracks containing background music were removed. Any screams that would have required more extensive editing were not used in the stimulus set. Finally, stimuli were matched for root-mean-square (RMS) amplitude to achieve a uniform average presentation volume across exemplars.

Three types of stimulus pairs were constructed, each consisting of two stimuli separated by a 2,000-ms interval of silence. Duration Modified pairs consisted of a scream and a transformed version of itself, identical in every parameter except duration, which in the transformed version was artificially lengthened or shortened using the Stretch function in Adobe Audition. The duration of the transformed scream ranged from 50 to $150 \%$ of the original stimulus duration $(M$ shortened $=71.78 \% ; M$ lengthened $=126.91 \%)$. These trials allowed us to gauge accuracy and response latency on what we assumed would be a relatively simpler version of the task, as vocalization duration, within limits, is not typically implicated as an indexical cue of identity-related characteristics such as body size (at least compared to frequency-related parameters; Pisanski et al., 2014). Same Vocalizer pairs consisted of two different exemplars produced by the same vocalizer; these either represented different screams within the same scream bout, or different screams from entirely separate bouts and eliciting contexts. Different 
208 Vocalizer pairs consisted of two exemplars produced by two different vocalizers, matched for 209 gender, relative age (e.g., child, adolescent, young adult, older adult), and apparent emotional context (including several cases in which a scream was matched with a foreign language-dubbed version produced for the exact same context). Figure 1 shows spectrograms of selected Same Vocalizer and Different Vocalizer pairs. trials, 24 Same Vocalizer trials, and 24 Different Vocalizer trials; this sample was comparable to that of a prior study demonstrating same-different identity discrimination for rhesus macaque screams (Fugate et al., 2008). Note that 21 out of 90 total exemplars (and 15 out of 47 total vocalizers) were re-used in combination with different screams; the numbers of unique exemplars and vocalizers in each condition are indicated in Table 1. During data preparation for analysis, two stimulus pairs — one Same Vocalizer pair and one Different Vocalizer pair — were each found to have been duplicated (i.e., the same two exemplars matched with one another, a result of mistakenly assigning audio files two different names). We omitted duplicate trials from analyses reported here, leaving 58 stimulus pairs (12 Duration Modified, 23 Same Vocalizer, and

22323 Different Vocalizer); however, we replicated all analyses using every possible combination of the duplicate stimulus pairs (e.g., the first Same Vocalizer pair and the second Different Vocalizer pair), and none of the study's major conclusions were affected. The few discrepancies — concerning participant factors related to variation-are noted in the

\section{Supplemental Results S1.}


231 between Duration Modified, Same Vocalizer, and Different Vocalizer trials (first exemplars: $F(2$,

$23255)=2.93, p=.062$; second exemplars: $F(2,55)=.81, p=.448)$, nor between female and male 233 screams (first exemplars: $t(56)=1.20, p=.235$; second exemplars: $t(56)=0.39, p=.699$ ). Of

234 the 58 stimulus pairs, 47 consisted of two acted screams (taken from film, television, or a sound

235 bank), 5 consisted of two naturally produced screams (taken from YouTube), and 6 consisted of 236 one acted scream and one natural scream.

237 Apparatus. The experiment was performed on a Sony VAIO Pentium 4 computer 238 (model PCV-RS311, Azumino, Japan). All sounds were presented through a pair of JVC G239 Series headphones (model HA-G55, JVCKENWOOD USA Corporation, Long Beach, CA).

240 Stimuli were delivered and data were collected using E-Prime 2.0 software (Psychology

241 Software Tools, Inc., Pittsburgh, PA). Participants provided input via a peripheral serial response 242 box (model 200a, Psychology Software Tools, Inc., Pittsburgh, PA); E-Prime records these 243 responses and their latencies with millisecond-precision timing.

244 Procedure

Discrimination task. Participants were asked to judge whether each pair of screams was produced by a single vocalizer ("Same") or two different vocalizers ("Different"), indicating their responses using two labelled buttons on the serial response box. A trial began when the word "Ready" appeared in the center of the screen. After a period of $500 \mathrm{~ms}$, the stimulus pair was delivered through the headphones while the screen displayed instructions reminding participants which button corresponded to which response. Participants were asked to respond as quickly and as accurately as possible, but only after the second scream in the pair had finished playing. When an input was received, an inter-trial interval of 1,000 ms proceeded with no visual

253 or auditory presentation. All participants heard the same 60 stimulus pairs, but trials were 
254 presented in a fully randomized order. An experimenter remained in another part of the testing

255 room to make sure instructions were followed, and to offer clarification on the task as necessary,

256 but otherwise no feedback was provided at any time.

257 Questionnaires. Following the discrimination task, personal and demographic

258 information was collected with the use of two self-report questionnaires. The first was a ten-item

259 survey developed specifically for studies in our lab. Seven items were designed to estimate the

260 participant's prior exposure to screams in the media, based on his or her agreement with

261 statements regarding the knowledge and consumption of video games, television, and film genres

262 likely to contain screams (e.g., "I watch TV shows where screaming often occurs"). All answers

263 were entered on a five-point scale, where higher ratings indicated greater knowledge or

264 familiarity. The remaining three items gauged the participant's confidence in making judgments

265 during the experiment and in reading emotions in general (e.g., "I consider myself good at

266 reading emotions in people”). Additionally, information regarding gender, age, first spoken

267 language, and handedness was collected (Supplemental Materials S1).

268 Empathy was measured using the Cambridge Behavior Scale (Baron-Cohen \&

269 Wheelwright, 2004), a 40-item questionnaire that assesses cognitive as well as emotional aspects

270 of empathic ability. The resultant Empathy Quotient (EQ) ranges from 0 to 80, with higher EQs

271 corresponding to greater levels of empathy.

272 Analysis

273 Overall accuracy and response latencies. Primary outcome variables derived from the

274 discrimination task included response accuracy (i.e., the proportion of accurate responses) and

275 the corresponding discriminability index $\left(d^{\prime}\right)$ for each participant. The $d^{\prime}$ 'statistic is used in

276 signal detection theory to estimate a participant's sensitivity to the difference between two 
277 different stimulus groups; it is an advantageous measure over percent accuracy because it

278 separates out the effects of a participant's response biases, which is especially important when

279 stimulus groups are not completely balanced (Macmillan \& Creelman, 2004). Each participant's

$280 d$ ' score was calculated as the difference between the Z-transformed Hit Rate and the Z-

281 transformed False Alarm Rate, where a Hit equated to correctly responding "Same" when the

282 two screams were produced by the same vocalizer (i.e., Duration Modified and Same Vocalizer

283 trials) and a False Alarm entailed incorrectly responding "Same" when two different vocalizers

284 had produced the screams (i.e., Different Vocalizer trials).

285 Mean response latencies were also calculated for each participant, using only the trials for

286 which the participant responded correctly (Bruyer \& Brysbaert, 2011). Responses provided

287 before the second exemplar had finished playing were not included in the analyses reported here,

288 as they were likely to reflect accidental or anticipatory inputs rather than decisions informed by

289 the full comparison of both exemplars. This resulted in the exclusion of 489 trials out of 6,032

290 trials across all participants. With two exceptions, noted in Supplemental Results S1, findings did

291 not differ for these analyses and a separate set in which the only exclusions were responses

292 entered before the second exemplar had started playing (precluding any comparison between the

293 exemplars; 26 exclusions).

294 Participant characteristics. A principal components analysis (PCA) with Varimax

295 rotation was conducted on the Spearman's correlation matrix obtained from participants'

296 responses to the questionnaire items. PCA was employed to reduce the number of variables-

297 thereby lowering the number of tests in subsequent regressions - because some of the items were

298 correlated with each other (likely due to probing the same underlying construct; O'Rourke \&

299 Hatcher, 2013). The analysis yielded 4 factors with Eigenvalues greater than 1, cumulatively 
300 explaining $70.78 \%$ of the variance in questionnaire responses (see Table 2). Based on the factor

301 loadings for each questionnaire item, our interpretation of each factor is as follows: F1 (Media)

302 captures exposure to media likely to contain screams, i.e., scary movies and television; F2

303 (Games) captures the tendency to play video games; F3 (Confidence) captures confidence in task

304 performance and in reading emotions generally; and F4 (Movies) captures exposure to movies

305 generally. To assess the effects of these factors on accuracy and response latency, participants'

306 factor scores were saved and used as predictor variables, along with EQ, in multiple linear

307 regressions with $d$ ' scores and reaction times as the outcome variables.

308 Statistical analyses were conducted using SPSS Statistics version 24 (IBM Corp.,

309 Armonk, NY). All statistical tests were two-tailed (where applicable) with a family-wise $\alpha=.05$.

310 RESULTS

311

312

313

314

315

316

317

\section{Discrimination Accuracies and Response Latencies: same or two different vocalizers}

The mean response accuracy across all participants and stimuli was .77 (SE: .01), corresponding to a mean $d^{\prime}$ score $=1.63\left(S E\right.$ : .06). In signal detection theory, $d^{\prime}=0$ indicates that participants failed to show discrimination on the tested perceptual dimension (i.e., discriminated at chance level), and an empirical threshold of $d^{\prime}>1.00$ is traditionally used to indicate that reliable discrimination was achieved (Macmillan \& Creelman, 2004; Fugate et al., 2008). Overall, participants achieved $d^{\prime}$ scores significantly above this criterion value $(t(103)=$ $11.02, p<.001)$, indicating that they discriminated between trials in which they heard the same vocalizer and trials in which they heard two different vocalizers.

Mean response accuracies and latencies were further analyzed by stimulus type. Onesample $t$-tests indicated that, on average, response accuracies exceeded chance level $(>.50)$ for each stimulus type (Duration Modified: $M=.95, S E=.01, t(103)=38.76, p<.001$; Same 
323 Vocalizer: $M=.76, S E=.01, t(103)=18.09, p<.001$; Different Vocalizer: $M=.71, S E=.01$,

$324 t(103)=14.15, p<.001)$. A nonparametric Friedman test (used due to non-normal distribution of

325 the Duration Modified data) with post-hoc, Bonferroni-corrected, Wilcoxon signed-rank

326 comparisons revealed that response accuracies varied significantly between stimulus types $\left(\chi^{2}(2)\right.$

$327=109.33, p<.001$; Figure 2): as we had expected, participants discriminated more accurately on

328 Duration Modified trials than on Same Vocalizer $(Z=8.30, p<.001)$ or Different Vocalizer trials

$329(Z=7.83, p<.001)$. Similarly, a repeated measures ANOVA revealed that mean response

330 latencies varied by stimulus type $\left(F(2,103)=30.57, p<.001, \eta_{\mathrm{p}}{ }^{2}=.229\right.$; Figure 3$)$ such that, on

331 trials in which participants responded correctly, they also reacted more quickly to Duration

332 Modified trials than to Same Vocalizer $(-264.69 \pm 33.86 \mathrm{~ms}, p<.001)$ or Different Vocalizer

333 trials $(-235.57 \pm 36.95 \mathrm{~ms}, p<.001)$. No significant difference was found between Same

334 Vocalizer and Different Vocalizer trials in either response accuracies $(p=.035$; not significant

335 with Bonferroni corrected $\alpha=.017)$ or latencies $(p=1.00)$.

336 Altogether, these results suggest that, as we had anticipated, it was easier for participants

337 to make accurate judgments on Duration Modified stimulus pairs (which, again, included a

338 scream and a lengthened or shortened version of itself) than on either of the other stimulus types.

339 In fact, 80 out of the 104 participants responded accurately on every Duration Modified stimulus

340 pair, suggesting a ceiling effect. We therefore recalculated response accuracies and $d$ ' scores

341 excluding Duration Modified trials to determine whether listeners could discriminate vocalizers

342 from unmodified screams. These calculations yielded a mean response accuracy $=.73(S E=.01)$

343 and a mean $d^{\prime}$ 'score $=1.40(S E=.06)$. Thus, although the overall discrimination level dropped

344 when only Same Vocalizer and Different Vocalizer trials were taken into account, it remained

345 significantly above the threshold value of $1.00(t(103)=6.80, p<.001)$, indicating that 
346 participants were able to discern whether unmodified scream exemplars where produced by the

347 same individual or by two different individuals.

348 Effects of Listener and Vocalizer Gender

349 Mixed factor ANOVAs were used to explore possible main effects and interactions

350 relating to listener and vocalizer gender. Vocalizer gender was used as a within-subjects factor

351 and listener gender was used as a between-subjects factor, with $d$ ' scores and mean response

352 latencies used as output variables in two separate tests. The results revealed a small main effect

353 of vocalizer gender on $d$ ' scores $\left(F(1,102)=4.10, p=.046, \eta_{\mathrm{p}}^{2}=0.039\right)$, suggesting that

354 listeners were better at discriminating male screams $(M=1.72, S E=.07)$ than female screams

$355(M=1.55, S E=.06)$. There was also a main effect of vocalizer gender on mean response

356 latencies $\left(F(1,102)=16.18, p<.001, \eta_{\mathrm{p}}{ }^{2}=.137\right)$, indicating that listeners responded more

357 slowly to male screams $(M=1,120.63, S E=57.44)$ than to female screams $(M=972.64, S E=$

358 43.93). No main effects of listener gender nor interacting effects of listener by vocalizer gender

359 were found for $d$ ' scores (listener gender: $p=.802$; interaction: $p=.492$ ) or response latencies

360 (listener gender: $p=.064$; interaction: $p=.498$ ).

361

Analyses were repeated excluding Duration Modified trials, revealing a slightly larger

362

main effect of vocalizer gender on $d^{\prime}$ scores within this subset $\left(F(1,102)=11.57, p<.001, \mathrm{\eta}_{\mathrm{p}}^{2}=\right.$

363.102 ; Figure 4), such that listeners discriminated more accurately when listening to male screams

$364(M=1.57, S E=.07)$ compared to female screams $(M=1.26, S E=.06)$. There also remained a

365 significant effect of vocalizer gender on mean response latencies $\left(F(1,102)=8.80, p=.004, \eta_{\mathrm{p}}{ }^{2}\right.$

$366=.079$; Figure 5) such that, when participants did respond accurately, they reacted more slowly

367 to male screams $(M=1,156.87 \mathrm{~ms}, S E=61.10)$ than to female screams $(M=1,033.64 \mathrm{~ms}, S E=$

368 47.24). No significant main effects of listener gender nor interacting effects of listener by 
369 vocalizer gender were found for $d$ ' scores (listener gender: $p=.641$; interaction: $p=.293$ ) or

370 response latencies (listener gender: $p=.057$; interaction: $p=.467$ ).

371 Effects of Other Participant Attributes

372 Multiple linear regressions were conducted to explore the relationship between

373 questionnaire-assessed participant attributes and their $d$ ' scores. Predictor variables included EQ

374 and the 4 factors extracted from a PCA on questionnaire responses (Media, Games, Confidence,

375 and Movies, Table 2). Two separate models were specified, one using the $d$ ' scores calculated

376 from responses across all trials, and one using the scores calculated with the exclusion of

377 Duration Modified trials. However, neither model significantly fit the data better than null,

378 intercept-only models (full dataset: $\mathrm{F}(5,98)=1.90, p=.101$; reduced dataset: $\mathrm{F}(5,98)=2.17, p$ $379=.063)$.

Multiple linear regressions were also conducted to examine the relationship between the

381

382

383

same predictor factors and mean reaction times on accurate responses in both the full dataset and the subset of data excluding Duration Modified trials. Again, neither model significantly fit the data better than null models (full dataset: $\mathrm{F}(5,98)=1.38, p=.238$; reduced dataset: $\mathrm{F}(5,98)=$ $1.26, p=.288)$.

\section{DISCUSSION}

Our results suggest that human screams permit a level of individual discrimination, such that listeners could determine whether two screams were produced by the same vocalizer or by two different vocalizers. Listeners responded accurately especially on trials in which they were presented a scream along with its modified version (Duration Modified trials); however, even when responses on these trials were excluded-leaving only screams that varied naturally within and between vocalizers (Same Vocalizer and Different Vocalizer trials, respectively) — mean 
392 response accuracies and $d$ ' scores significantly exceeded chance levels, suggesting that screams

393 can convey sufficient information to discriminate individuals.

Previous research using isolated vowel sounds and laughter has shown that cues unique

395

396

397

398

399

400

401

402

403

404

405

406

407

408

409

410

411

412

413

414

to speech per se are not required for the perception of vocal identity in humans (Gaudrain, Ban, \& Patterson, 2009; Kuwabara \& Takagi, 1991; Lavan et al., 2016; Owren \& Rendall, 2003). Our results extend this work by demonstrating that identity-related vocal qualities are perceived in at least some screams as well. This finding is inconsistent with the results of Hansen et al. (2017), who reported that listeners discriminated at chance levels in a same-different identity discrimination task using screams. That study used a very small sample size and did not account for potential heterogeneity in the data relating to participant ability or to the screams themselves. Our results are more in line with evidence that non-human primate screams, which share many of the acoustic characteristics of human screams, enable receivers to assess and respond to a caller's identity (Cheney \& Seyfarth, 1980; Fugate et al., 2008; Seyfarth \& Cheney 2015), suggesting that this informative capacity is one common to human and non-human screams.

We did not observe a main effect nor any interacting effects relating to listener gender. Our relatively small sample of male participants and vocalizers might have limited the power of these tests. However, the results did show significant variability relating to vocalizer gender: male screams were more accurately discriminated than female screams. Although this effect was marginal in the full dataset, it emerged clearly in the subset of data taken from Same Vocalizer and Different Vocalizer trials, i.e., when the ceiling effect of Duration Modified trials was removed. We had predicted that gender effects might account for variation in this task because they also explain some variation in recognizing emotion from vocalizations (Belin, FillionBilodeau, \& Gosselin, 2008; Lausen \& Schacht, 2018). If anything, however, evidence suggests 
415 that females are better than males at expressing emotion through nonlinguistic vocalizations such

416 as affect bursts (Lausen \& Schacht, 2018), so it seems unlikely that the advantage conferred by

417 male vocalizers here is related to enhanced emotional expression.

418 A possible alternative explanation for the vocalizer gender finding was proposed by

419 Owren and Rendall (2003), who also reported that listeners were better at discriminating identity

420 when responding to male versus female exemplars, a finding they attributed to the lower

421 fundamental frequencies (F0) and consequently richer harmonic spectra of male vocalizations. A

422 similar proximate mechanism may be at work in this study, but we note that findings regarding

423 sex differences in vocal communication may merit consideration of evolutionary issues as well.

424 Screams are evidently more widely produced by females than by males (Anikin \& Persson,

425 2017), a tendency we share with some nonhuman primates (e.g., Bernstein \& Ehardt, 1985). One

426 possibility, then, given the relative rarity of male screams, is that they differ from female screams

427 with respect to salience to listeners, a quality that in turn could affect listeners' interpretations.

428 Although it is difficult to evaluate this explanation here, particularly because female screams

429 were discriminated more quickly than male screams, further exploration of sex and gender

430 differences in screams is likely to prove a fruitful avenue of research.

We did not find strong evidence in analyses of either accuracies or response times to

432 support the hypothesis that prior experience with screams would facilitate the perception and use

433 of identity cues. It is possible, instead, that experience with a specific individual's vocal

434 qualities, rather than experience with the vocal class as a whole, would enable better

435 discrimination from that individual's screams, as has been demonstrated in laughter (Lavan et al.,

436 2016) and speech (Schmidt-Nielsen \& Stern, 1985). We also note that our questionnaire only

437 assessed experience with screams in the media. Future research might therefore explore broader 
438 measures of experience with screams, including those relating to personal familiarity with the

439 vocalizers as well as exposure to screams in real-life scenarios.

Additionally, we did not find evidence that differences in empathy accounted for

441 variation in participants' abilities to discriminate identity. Although empathy likely plays a role

442 in motivating the decision to respond to screams (de Waal, 2008), it is plausibly less involved at

443 the stage of identity perception. Given that our hypothesis with respect to listener gender was

444 also not borne out - any gender differences in emotional processing did not translate into gender

445 differences on this task - these results may suggest that the recognition of identity is a process

446 somewhat independent from that of recognizing or experiencing the emotional aspects of

447 screams, consistent with models of voice perception that posit separate functional pathways for

448 the processing of vocal identity and affect (Belin, Fecteau, and Bédard, 2004). More research is

449 needed, however, to test this hypothesis specifically in screams.

$450 \quad$ Owren and Rendall (2003) contended that screams are not ideal for communicating

451 identity in part because they lack the stable, tightly-spaced harmonics of some other

452 vocalizations. They argued that the distribution of energy across a rich, tonal harmonic spectrum

453 is important for revealing the individually distinctive effects of supralaryngeal filtering.

454 Consistent with this claim, a same-different identity discrimination task in their study using the

455 spoken vowel sound / $€ /$ - the acoustic profile of which is marked by closely stacked

456 harmonics - yielded a mean $d$ ' score of 3.5 (Owren \& Rendall, ibid.), much higher than the

457 average scores observed in our experiment. However, increasing evidence suggests that vocal

458 cues to identity in humans are redundant (Mathias \& von Kriegstein, 2014). That supralaryngeal

459 filter effects seem important for individual recognition from tonal vocalizations does not

460 necessarily mean that vocalizations where filter effects are masked will lack cues to identity. 
461 Indeed, humans can recognize vocalizers from speech even when all harmonics except F0 (a

462 parameter related to source vibrations rather than the filter) have been eliminated (Abberton \&

463 Fourcin, 1978), and research in other nonlinguistic vocalizations suggests that F0 as well as

464 spectral characteristics may vary systematically between individuals (e.g., in infant cries;

465 Gustafsson et al., 2013). Another intriguing possibility in screams is that nonlinear phenomena

466 such as chaotic noise and bifurcations between harmonic regimes might serve as additional

467 identity cues (Fitch, Neubauer, \& Herzel, 2002). The role of these acoustic parameters in

468 communicating identity in screams is a promising question for future research.

469 One issue that has received increased attention in recent literature is that of potential

470 differences between acted and naturally occurring nonlinguistic vocalizations (Anikin \& Lima,

471 2017; Anikin \& Persson, 2017; Bryant et al., 2018; Engelberg \& Gouzoules, 2018). Of particular

472 relevance here, Lavan et al. (2018b) found that listeners' abilities to discriminate identity from

473 laughter varied as a function of vocal production mode, i.e., whether the laughter was

474 spontaneous or volitional. In that study, participants' discrimination abilities were significantly

475 impaired when presented with spontaneous laughter; indeed, their $d$ ' scores fell below those

476 obtained from the present experiment. Given that most screams in our study were produced by

477 professional actors, these findings in laughter raise the possibility that our participants may also

478 have discriminated less accurately had we incorporated more naturally produced screams. For

479 example, acted screams are possibly more stereotyped than natural ones within an individual,

480 which could facilitate the matching of two exemplars produced by the same vocalizer. That said,

481 our previous study regarding this issue showed that actors are capable of producing credible

482 screams (Engelberg \& Gouzoules, 2018). An additional possibility is that the lower $d$ 'scores for

483 spontaneous laughter relative to screams reflects the inherent differences of two distinct vocal 
484 classes. Laughter is a highly acoustically variable vocalization (Bachorowski, Smoski, \& Owren,

485 2001), which may hinder listeners' abilities to recognize an individual. We have begun to

486 characterize the acoustic variation within screams (Schwartz, Engelberg, \& Gouzoules, under

487 review), but the full extent of this variation within individuals remains to be established. Potential confounds in our experiment might include any acoustic variation in Different Vocalizer pairs introduced from sources other than individual identity, such as differences in the emotional contexts of screams or the age of vocalizers. Although we matched both of these factors within stimulus pairs to the fullest extent possible, exact matches (e.g., for the precise age of every vocalizer) were not always feasible. Conceivably, slight differences in vocalizer age might have contributed to listeners' judgments, as the mean age difference in Different Vocalizer pairs would exceed that of Same Vocalizer pairs. That said, we know of no empirical evidence in the literature to suggest that listeners can assess age from screams, especially with the degree of precision necessary to steer participants' responses on our task. Likewise, although our sample 497 size precluded context-based analyses, it would be of interest for future research to account for the role of emotional context in identity perception from screams, particularly because screams are so contextually heterogeneous (spanning contexts of fear, anger, joy, and pain; Anikin \& Persson, 2017). One open question concerns the extent to which identity cues are contextspecific as opposed to consistent across contextually disparate screams. Ongoing and planned investigations in our lab, exploring the acoustic and perceptual differences between screams 503 produced in different emotional contexts, may shed some light on these issues. 
A final potential if unlikely confound is the possibility of learning effects. Given that 21

507

508

509

510

511

512

513

514

515

516

517

518

519

520

521

522

523

524

525

526

527

528

exemplars and 15 vocalizers appeared in multiple stimulus pairs, listeners may have benefited

from hearing the same scream more than once across the experiment. However, we suggest that

if any such benefits occurred, listeners would likely have learned cues distinctive to the repeated vocalizers, consistent with the conclusion that screams convey identity cues.

It is important to note a distinction between the discrimination tested here and true individual recognition. Our study required that participants listen to two consecutively presented exemplars and decide whether they were produced by the same vocalizer or different vocalizers; recognition entails matching a perceived vocalization against a stored, cognitive representation of a familiar individual's vocal attributes (Sidtis \& Krieman, 2012; Steiger \& Müller, 2008;

Tibbetts \& Dale, 2007). When a non-human primate in a playback experiment responds preferentially to her offspring's screams (Cheney \& Seyfarth, 1980; Gouzoules et al., 1986), she is likely demonstrating recognition at some level, especially because many such studies present listeners only one call or short bout within a span of several days (Cheney \& Seyfarth, 1980).

Our results demonstrate a prerequisite to this kind of vocal recognition, in that human screams are sufficiently distinct between vocalizers to permit immediate discrimination, but further research is needed to determine whether listeners could identify a scream as belonging to a particular individual. Additional questions concern the mechanisms underlying individual discrimination from screams and the processes involved in the development of these abilities.

\section{CONCLUSIONS}

The finding that human screams convey cues to screamer identity is consistent with the wealth of evidence for a similar capacity in nonhuman primates (Bergman et al., 2003; Cheney \& Seyfarth, 1980; Fugate et al., 2008; Gouzoules et al., 1986; Kojima et al., 2003; Seyfarth \& 
529 Cheney, 2015; Slocombe et al., 2010). Nonetheless, this question has been the subject of

530 considerable and ongoing debate (Fugate et al., 2008; Owren \& Rendall, 2003; Rendall et al.,

531 1998). The present study contributes to this area of research by demonstrating an ability among

532 humans to discriminate between individual screamers based on their screams alone. This result

533 supports evolutionary continuity in at least some aspects of scream function between humans and

534 other primate species. In general, more is known about the functions of screams in nonhuman

535 primates than in humans; more research is needed in order to elucidate which aspects of human

536 scream production and perception are evolutionarily shared and which are derived.

537 ACKNOWLEDGEMENTS

538 We thank Caitlin Clark, Alexander Gouzoules, Leah Friedman, Elizabeth Harlan, and

539 NooRee Lee for assistance with stimulus collection, as well as Anna Duncan for assistance with

540 data collection. 


\section{REFERENCES}

Abberton, E., \& Fourcin, A. J. (1978). Intonation and speaker identification. Language and Speech, 21(4), 305-318.

Anikin, A., Bååth, R., \& Persson, T. (2018). Human non-linguistic vocal repertoire: Call types and their meaning. Journal of Nonverbal Behavior, 42(1), 53-80.

Anikin, A., \& Lima, C. F. (2017). Perceptual and acoustic differences between authentic and acted nonverbal emotional vocalizations. The Quarterly Journal of Experimental Psychology, 1-21.

Anikin, A., \& Persson, T. (2017). Nonlinguistic vocalizations from online amateur videos for emotion research: A validated corpus. Behavior Research Methods, 49(2), 758-771.

Arnal, L. H., Flinker, A., Kleinschmidt, A., Giraud, A. L., \& Poeppel, D. (2015). Human screams occupy a privileged niche in the communication soundscape. Current Biology, 25(15), 2051-2056.

Bachorowski, J. A., Smoski, M. J., \& Owren, M. J. (2001). The acoustic features of human laughter. The Journal of the Acoustical Society of America, 110(3), 1581-1597.

Beecher, M. D. (1990). The evolution of parent-offspring recognition in swallows. In D. A. Dewsbury (Ed.), Contemporary issues in comparative psychology (pp. 360-380). Sunderland, MA, US: Sinauer Associates.

Belin, P. (2006). Voice processing in human and non-human primates. Philosophical Transactions of the Royal Society of London B: Biological Sciences, 361(1476), 20912107.

Belin, P., Fecteau, S., \& Bedard, C. (2004). Thinking the voice: neural correlates of voice perception. Trends in Cognitive Sciences, 8(3), 129-135. 
Belin, P., Fillion-Bilodeau, S., \& Gosselin, F. (2008). The Montreal Affective Voices: a validated set of nonverbal affect bursts for research on auditory affective processing. Behavior Research Methods, 40(2), 531-539.

Bergman, T. J., Beehner, J. C., Cheney, D. L., \& Seyfarth, R. M. (2003). Hierarchical classification by rank and kinship in baboons. Science, 302(5648), 1234-1236.

Bernstein, I. S., \& Ehardt, C. L. (1985). Age-sex differences in the expression of agonistic behavior in rhesus monkey (Macaca mulatta) groups. Journal of Comparative Psychology, 99(2), 115.

Bruyer, R., \& Brysbaert, M. (2011). Combining speed and accuracy in cognitive psychology: Is the inverse efficiency score (IES) a better dependent variable than the mean reaction time (RT) and the percentage of errors (PE)? Psychologica Belgica, 51(1), 5-13.

Bryant, G. A., \& Aktipis, C. A. (2014). The animal nature of spontaneous human laughter. Evolution and Human Behavior, 35(4), 327-335.

Bryant, G. A., Fessler, D. M., Fusaroli, R., Clint, E., Amir, D., Chávez, B., ... \& Fux, M. (2018). The perception of spontaneous and volitional laughter across 21 societies. Psychological Science, 29(9), 1515-1525.

Charrier, I., Pitcher, B. J., \& Harcourt, R. G. (2009). Vocal recognition of mothers by Australian sea lion pups: individual signature and environmental constraints. Animal Behaviour, 78(5), 1127-1134.

Cheney, D. L. (1977). The acquisition of rank and the development of reciprocal alliances among free-ranging immature baboons. Behavioral Ecology and Sociobiology, 2(3), 303-318.

Cheney, D. L., \& Seyfarth, R. M. (1980). Vocal recognition in free-ranging vervet monkeys. 
Animal Behaviour, 28(2), 362-367.

Davila-Ross, M. D., Owren, M. J., \& Zimmermann, E. (2010). The evolution of laughter in great apes and humans. Communicative \& Integrative Biology, 3(2), 191-194.

de Waal, F. B., \& Van Hooff, J. A. (1981). Side-directed communication and agonistic interactions in chimpanzees. Behaviour, 77(3), 164-198.

de Waal, F. B. (2008). Putting the altruism back into altruism: the evolution of empathy. Annual Review of Psychology, 59, 279-300.

Donohue, S. E., Woldorff, M. G., \& Mitroff, S. R. (2010). Video game players show more precise multisensory temporal processing abilities. Attention, Perception, \& Psychophysics, 72(4), 1120-1129.

Engelberg, J. W., \& Gouzoules, H. (2018). The credibility of acted screams: Implications for emotional communication research. Quarterly Journal of Experimental Psychology. doi $10.1177 / 1747021818816307$

Fitch, W. T. (1997). Vocal tract length and formant frequency dispersion correlate with body size in rhesus macaques. The Journal of the Acoustical Society of America, 102(2), $1213-1222$.

Fitch, W. T., Neubauer, J., \& Herzel, H. (2002). Calls out of chaos: The adaptive significance of nonlinear phenomena in mammalian vocal production. Animal Behaviour, 63(3), 407418.

Fugate, J., Gouzoules, H., \& Nygaard, L. C. (2008). Recognition of rhesus macaque (Macaca mulatta) noisy screams: evidence from conspecifics and human listeners. American Journal of Primatology, 70(6), 594-604.

Gaudrain, E., Li, S., Ban, V. S., \& Patterson, R. D. (2009). The role of glottal pulse rate and 
vocal tract length in the perception of speaker identity. In Tenth Annual Conference of the International Speech Communication Association, Brighton, United Kingdom, pp. $148-151$.

Gonzalez, J., \& Oliver, J. C. (2005). Gender and speaker identification as a function of the number of channels in spectrally reduced speech. The Journal of the Acoustical Society of America, 118(1), 461-470.

Gouzoules, H. (2005) Monkeying Around with Symbolism: Are Vocalizations Simple Symbols...or More Like Cymbals? In L. Namy, Laura. (Ed). Symbol use and symbolic representation: Developmental and comparative perspectives. (pp. 245-265). Mahwah, NJ: Lawrence Erlbaum Associates Publishers.

Gouzoules, H., \& Gouzoules, S. (2000). Agonistic screams differ among four species of macaques: the significance of motivation-structural rules. Animal Behaviour, 59(3), 501512.

Gouzoules, H., Gouzoules, S., \& Marler, P. (1986). Vocal communication: a vehicle for the study of social relationships. In R. Rawlins \& M. Kessler. (Eds.), The Cayo Santiago macaques: History, behavior, and biology (pp. 111-129). Albany, NY: SUNY Press.

Gouzoules, S., Gouzoules, H., \& Marler, P. (1984). Rhesus monkey (Macaca mulatta) screams: representational signalling in the recruitment of agonistic aid. Animal Behaviour, 32(1), 182-193.

Green, C. S., \& Bavelier, D. (2012). Learning, attentional control, and action video games. Current Biology, 22(6), R197-R206.

Green, J. A., \& Gustafson, G. E. (1983). Individual recognition of human infants on the basis of cries alone. Developmental Psychobiology, 16(6), 485-493. 
Gustafson, E., Levréro, F., Reby, D., \& Mathevon, N. (2013). Fathers are just as good as mothers at recognizing the cries of their baby. Nature Communications, 4, 1698.

Gustafson, G. E., Green, J. A., \& Tomic, T. (1984). Acoustic correlates of individuality in the cries of human infants. Developmental Psychobiology, 17(3), 311-324.

Hansen, J. H., Nandwana, M. K., \& Shokouhi, N. (2017). Analysis of human scream and its impact on text-independent speaker verification. The Journal of the Acoustical Society of America, 141(4), 2957-2967.

Knörnschild, M., Feifel, M., \& Kalko, E. K. (2013). Mother-offspring recognition in the bat Carollia perspicillata. Animal Behaviour, 86(5), 941-948.

Kojima, S., Izumi, A., \& Ceugniet, M. (2003). Identification of vocalizers by pant hoots, pant grunts and screams in a chimpanzee. Primates, 44(3), 225-230.

Kuwabara, H., \& Takagi, T. (1991). Acoustic parameters of voice individuality and voicequality control by analysis-synthesis method. Speech Communication, 10(5-6), 491-495.

Lausen, A., \& Schacht, A. (2018). Gender differences in the recognition of vocal emotions. Frontiers in Psychology, 9, 882.

Lavan, N., Burton, A.M., Scott, S.K., \& McGettigan, C. (2018a). Flexible voices: Identity perception from variable vocal signals. Psychonomic Bulletin \& Review. Published online. https://doi.org/10.3758/s13423-018-1497-7

Lavan, N., Scott, S. K., \& McGettigan, C. (2016). Impaired generalization of speaker identity in the perception of familiar and unfamiliar voices. Journal of Experimental Psychology: General, 145(12), 1604.

Lavan, N., Short, B., Wilding, A., \& McGettigan, C. (2018b). Impoverished encoding of speaker identity in spontaneous laughter. Evolution and Human Behavior, 39(1), 139- 
145.

Lingle, S., Rendall, D., \& Pellis, S. M. (2007). Altruism and recognition in the antipredator defence of deer: 1. Species and individual variation in fawn distress calls. Animal Behaviour, 73(5), 897-905.

Lingle, S., Wyman, M. T., Kotrba, R., Teichroeb, L. J., \& Romanow, C. A. (2012). What makes a cry a cry? A review of infant distress vocalizations. Current Zoology, 58(5), 698-726.

Macmillan, N. A., \& Creelman, C. D. (2004). Detection theory: A user's guide. Cambridge, United Kingdom: Cambridge University Press.

Mathias, S. R., \& von Kriegstein, K. (2014). How do we recognise who is speaking. Frontiers in Bioscience (Scholar Edition), 6, 92-109.

McCune, L., Vihman, M. M., Roug-Hellichius, L., Delery, D. B., \& Gogate, L. (1996). Grunt communication in human infants (Homo sapiens). Journal of Comparative Psychology, $110(1), 27$.

O'Rourke, N., \& Hatcher, L. (2013). A step-by-step approach to using SAS for factor analysis and structural equation modeling. Cary, NC: SAS Institute.

Owren, M. J., \& Bachorowski, J.-A. (2007). Measuring emotion-related vocal acoustics. In J. Coan, \& J. Allen (Eds.), Handbook of emotion elicitation and assessment (pp. 239-266). Oxford, England: Oxford University Press.

Owren, M. J., \& Rendall, D. (2003). Salience of caller identity in rhesus monkey (Macaca mulatta) coos and screams: perceptual experiments with human (Homo sapiens) listeners. Journal of Comparative Psychology, 117(4), 380.

Owren, M. J., Amoss, R. T., \& Rendall, D. (2011). Two organizing principles of vocal 
production: Implications for nonhuman and human primates. American Journal of Primatology, 73(6), 530-544.

Perrachione, T. K., Del Tufo, S. N., \& Gabrieli, J. D. (2011). Human voice recognition depends on language ability. Science, 333(6042), 595-595.

Pisanski, K., Fraccaro, P. J., Tigue, C. C., O'Connor, J. J., Röder, S., Andrews, P. W., ... \& Feinberg, D. R. (2014). Vocal indicators of body size in men and women: a metaanalysis. Animal Behaviour, 95, 89-99.

Raine, J., Pisanski, K., Oleszkiewicz, A., Simner, J., \& Reby, D. (2018). Human listeners can accurately judge strength and height relative to self from aggressive roars and speech. iScience, 4, 273-280.

Remez, R. E., Fellowes, J. M., \& Rubin, P. E. (1997). Talker identification based on phonetic information. Journal of Experimental Psychology: Human Perception and Performance, $23(3), 651$.

Rendall, D., Owren, M. J., \& Rodman, P. S. (1998). The role of vocal tract filtering in identity cueing in rhesus monkey (Macaca mulatta) vocalizations. The Journal of the Acoustical Society of America, 103(1), 602-614.

Rendall, D., Notman, H., \& Owren, M. J. (2009). Asymmetries in the individual distinctiveness and maternal recognition of infant contact calls and distress screams in baboons. The Journal of the Acoustical Society of America, 125(3), 1792-1805.

Rohwer, S., Fretwell, S. D., \& Tuckfield, R. C. (1976). Distress screams as a measure of kinship in birds. American Midland Naturalist, 418-430.

Seyfarth, R. M., \& Cheney, D. M. (2015). The Evolution of Concepts about Agents: Or, What 
Do Animals Recognize When They Recognize an Individual? In E. Margolis, \& S.

Laurence (Eds.), The conceptual mind: New directions in the study of concepts (pp. 57-

75). Cambridge, MA: MIT Press.

Schwartz, J., Engelberg, J., \& Gouzoules, H. (2019). What is a scream? Listener agreement and acoustic characteristics. Under review.

Sheffert, S. M., Pisoni, D. B., Fellowes, J. M., \& Remez, R. E. (2002). Learning to recognize talkers from natural, sinewave, and reversed speech samples. Journal of Experimental Psychology: Human Perception and Performance, 28(6), 1447.

Sidtis, D., \& Kreiman, J. (2012). In the beginning was the familiar voice: personally familiar voices in the evolutionary and contemporary biology of communication. Integrative Psychological and Behavioral Science, 46(2), 146-159.

Silk, J. B. (2002). Kin selection in primate groups. International Journal of Primatology, 23(4), 849-875.

Slocombe, K. E., Kaller, T., Call, J., \& Zuberbühler, K. (2010). Chimpanzees extract social information from agonistic screams. PLoS One, 5(7), e11473.

Steiger, S., \& Müller, J. K. (2008). 'True' and 'untrue' individual recognition: suggestion of a less restrictive definition. Trends in ecology \& evolution, 23(7), 355.

Taylor, A. M., \& Reby, D. (2010). The contribution of source-filter theory to mammal vocal communication research. Journal of Zoology, 280(3), 221-236.

Tibbetts, E. A., \& Dale, J. (2007). Individual recognition: it is good to be different. Trends in Ecology \& Evolution, 22(10), 529-537.

Van Lancker, D., Kreiman, J., \& Emmorey, K. (1985). Familiar voice recognition: Patterns and parameters: I. Recognition of backward voices. Journal of Phonetics, 13, 19-38. 
Wickelgren, W. A. (1977). Speed-accuracy tradeoff and information processing dynamics. Acta Psychologica, 41(1), 67-85.

Wiesenfeld, A. R., Whitman, P. B., \& Malatesta, C. Z. (1984). Individual differences among adult women in sensitivity to infants: evidence in support of an empathy concept. Journal of Personality and Social Psychology, 46(1), 118.

Yorzinski, J. L. (2017). The cognitive basis of individual recognition. Current Opinion in Behavioral Sciences, 16, 53-57. 


\section{Table $\mathbf{1}$ (on next page)}

Stimulus pair information.

Breakdown of vocalizer sex as well as the numbers of unique exemplars and unique vocalizers for each stimulus type. Transformed versions of screams are counted as unique exemplars in the Duration Modified pairs. The total numbers of unique vocalizers and exemplars are less than the sum of their columns because some exemplars are repeated between but not within stimulus types. 
Table 1

\begin{tabular}{|c|c|c|c|c|c|}
\hline & Female & Male & Unique & Unique & Number of \\
\hline Stimulus Type & $\underline{\text { Exemplars }}$ & Exemplars & Exemplars & $\underline{\text { Vocalizers }}$ & $\underline{\text { Pairs }}$ \\
\hline Duration Modified & 10 & 2 & 24 & 12 & 12 \\
\hline Same Vocalizer & 16 & 7 & 40 & 14 & 23 \\
\hline Different Vocalizer & 15 & 8 & 40 & 35 & 23 \\
\hline Total & 41 & 17 & 90 & 47 & 58 \\
\hline
\end{tabular}

1

2 
Table 2 (on next page)

Results of PCA on questionnaire responses.

(A) Rotated components matrix with factor loadings. (B) Summary of PCA results including all PCs with Eigenvalues $>1$. 
A. Rotated Components Matrix from PCA on Questionnaire Responses

\begin{tabular}{lrrrr}
\hline $\begin{array}{lrrr}\text { Questionnaire Item } \\
\text { Watch movies }\end{array}$ & $\underline{F}$ & $\underline{F} 2$ & $\underline{F}$ & $\underline{F}$ \\
Enjoy scary movies & 0.105 & 0.05 & -0.154 & $\mathbf{0 . 8 5 6}$ \\
Watch scary movies & $\mathbf{0 . 8 7 6}$ & 0.052 & 0.137 & -0.023 \\
Watch TV with screams & $\mathbf{0 . 8 3 9}$ & 0.134 & 0.135 & 0.118 \\
Know about movie actors & $\mathbf{0 . 7 2 8}$ & -0.049 & 0.007 & 0.143 \\
Know about video games & 0.103 & -0.059 & 0.208 & $\mathbf{0 . 8 3 8}$ \\
Play action video games & 0.047 & $\mathbf{0 . 9 5 3}$ & 0.064 & 0.003 \\
Confidence about judgments & 0.059 & $\mathbf{0 . 9 4 3}$ & 0.14 & -0.01 \\
Difficulty making choices & -0.035 & 0.197 & $\mathbf{0 . 7 2 5}$ & -0.003 \\
Reading emotions & 0.089 & -0.132 & $\mathbf{0 . 6 1 3}$ & 0.079
\end{tabular}

1

2 Note. Factor loadings $>0.4$ are bolded.

3

B. Summary of PCA Results on Questionnaire Responses

$\underline{\text { Factor }}$

Eigenvalue

explained (\%)

2.708

27.08

18.84

13.11

11.76

$\underline{\text { Cumulative variance }}$

explained (\%)

F1. Media

1.884

1.311

1.176

F4. Movies 
Figure 1

Selected examples of scream pairs.

Same Scores (the proportion of participates who indicated that the pair came from the same vocalizer) noted in percentiles. (A) scream pairs by a single vocalizer that yielded high Same Scores; (B) scream pairs by different vocalizers that yielded high Same Scores; (C) scream pairs by a single vocalizer that yielded low Same Scores; (D) scream pairs by different vocalizers that yielded low Same Scores. 
A
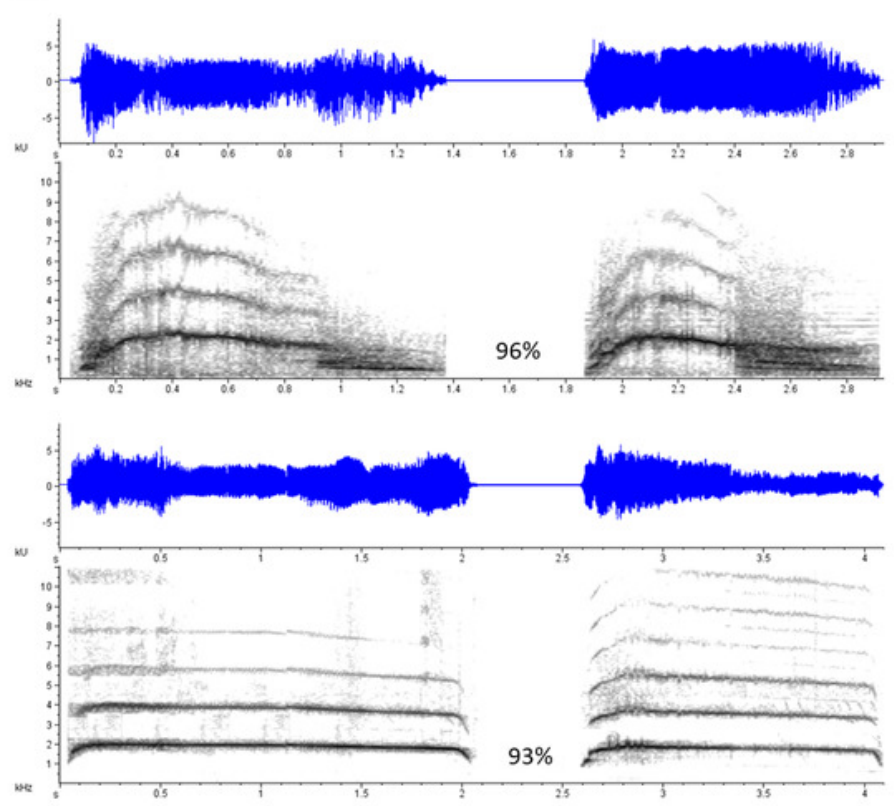

C
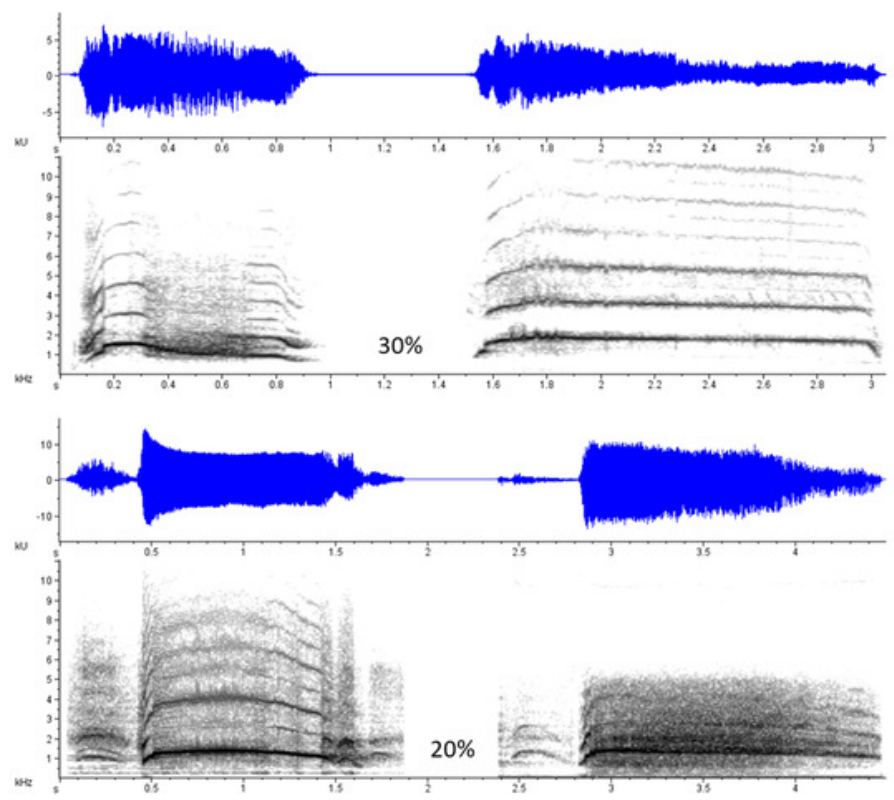

B
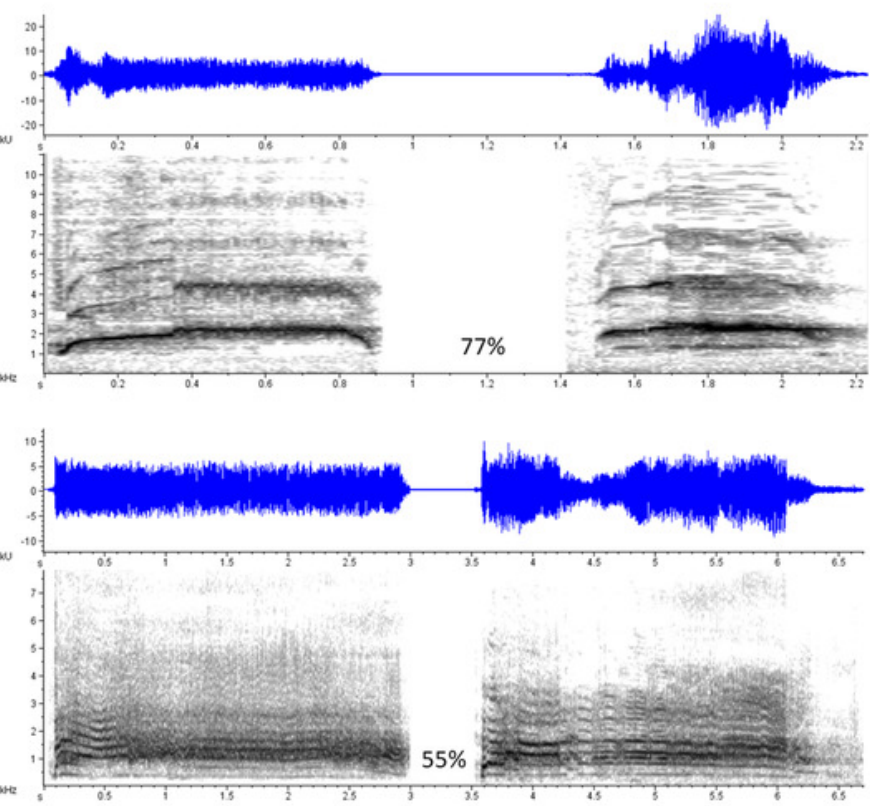

D
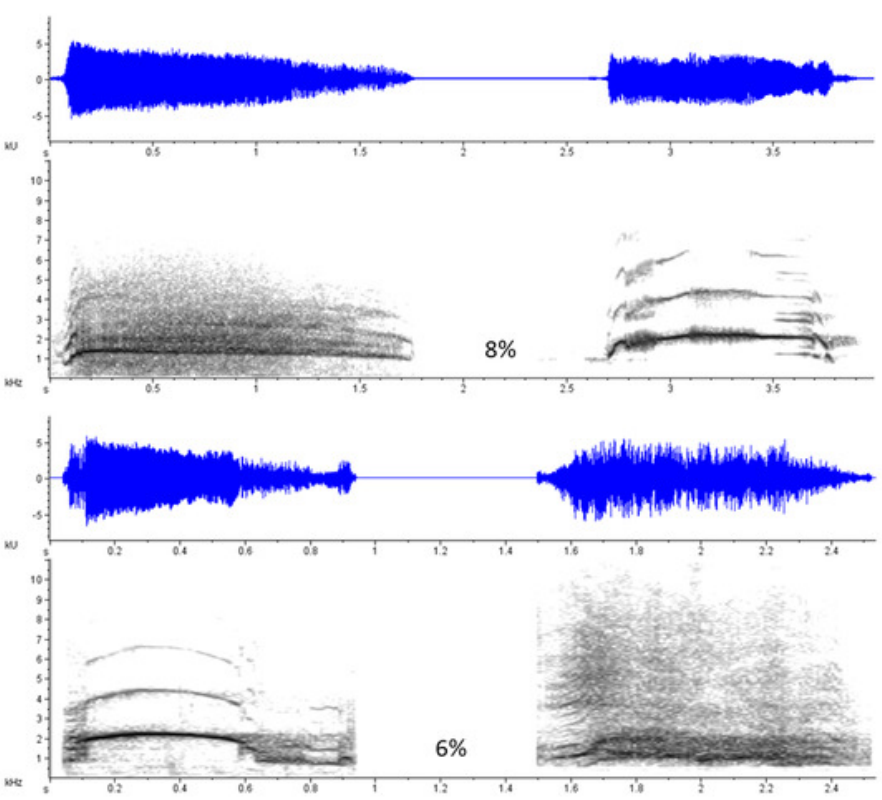
Figure 2

Minimum, quartiles, and maximum response accuracies for Duration Modified, Same Vocalizer, and Different Vocalizer stimulus types; $X$ represents group means.

Asterisk indicates significance, $p<.05$, with the following numbers indicating the estimate of the true difference of the means and the $95 \%$ confidence intervals.

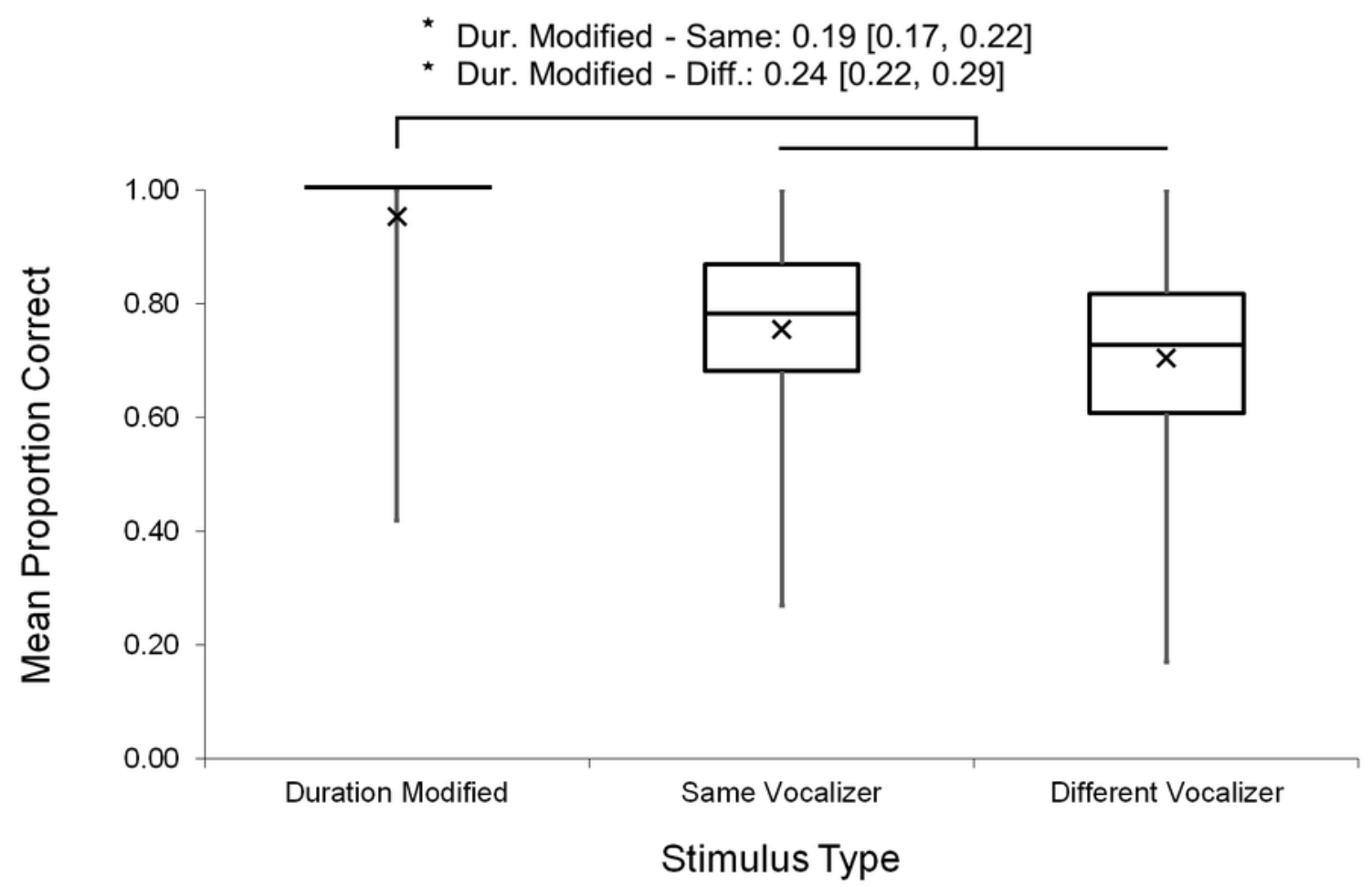


Figure 3

Minimum, quartiles, and maximum response latencies on accurate responses for Duration Modified, Same Vocalizer, and Different Vocalizer stimulus types; $X$ represents group means.

Asterisk indicates significance, $p<.05$, with the following numbers indicating the estimate of the true difference of the means and the $95 \%$ confidence intervals.

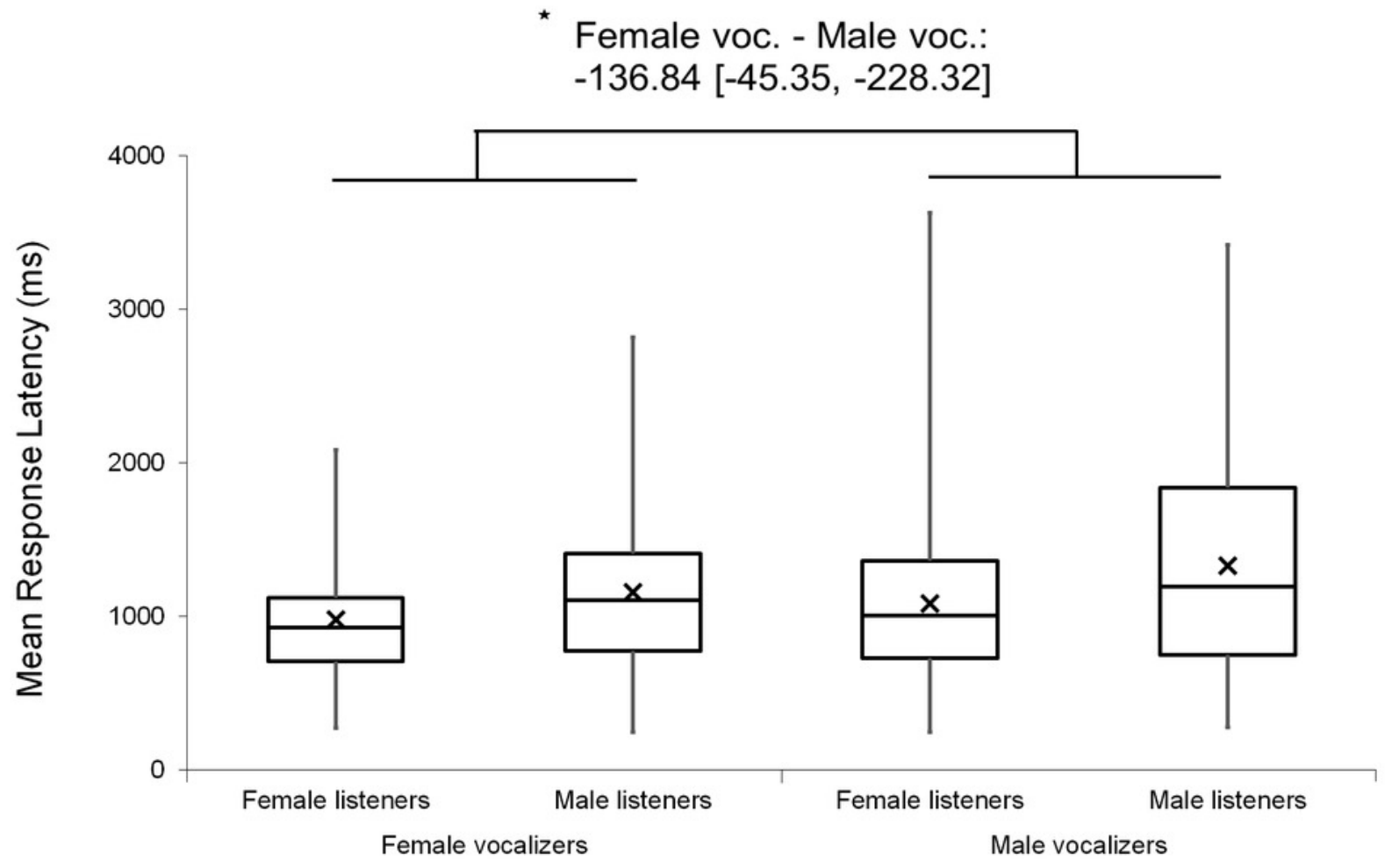


Figure 4

Minimum, quartiles, and maximum sensitivity indices ( $d^{\prime}$ scores) by listener gender and vocalizer gender; $X$ represents group means.

Duration Modified stimuli were omitted for this graph. Asterisk indicates significance, $p<.05$, with the following numbers indicating the estimate of the true difference of the means and the $95 \%$ confidence intervals.

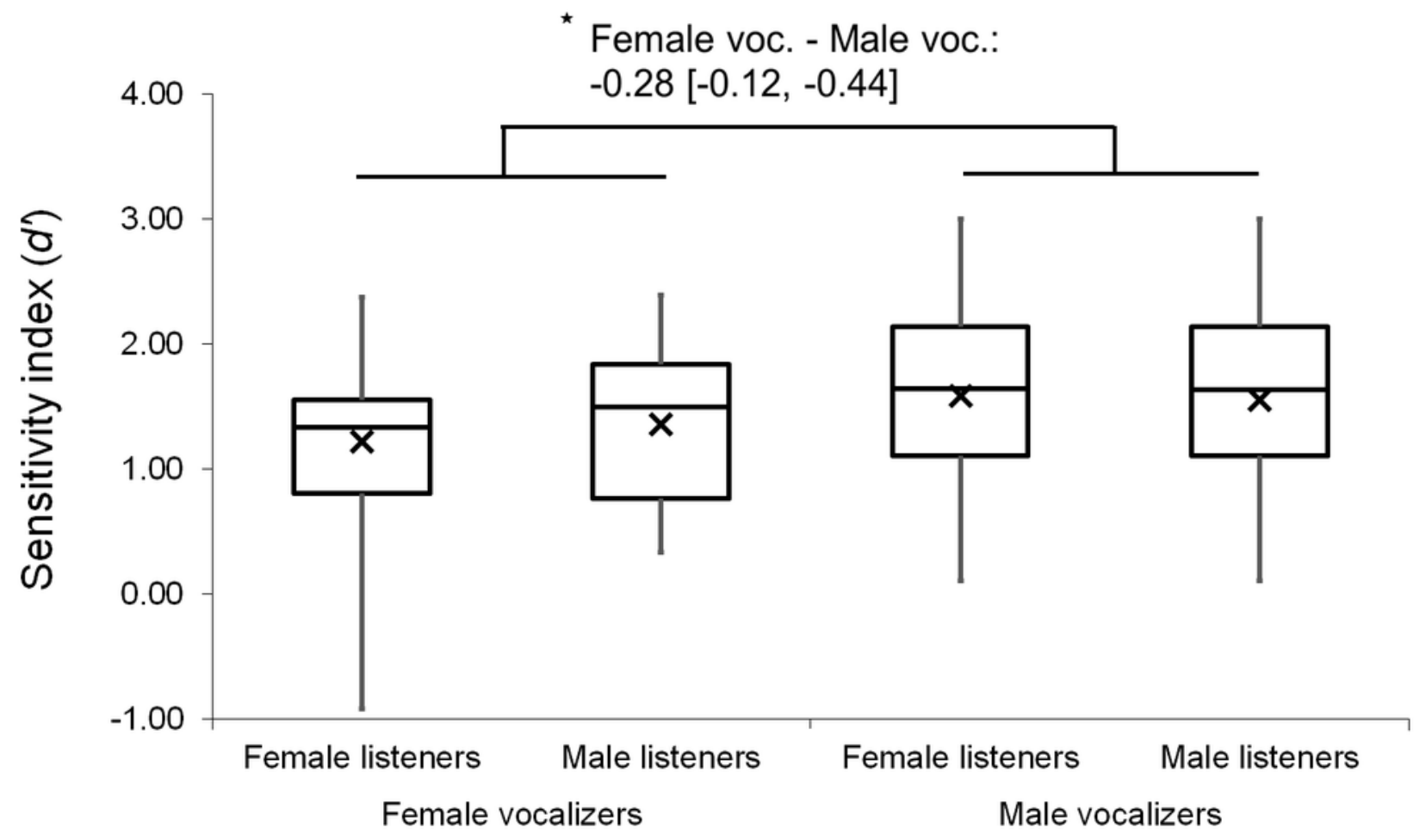


Figure 5

Minimum, quartiles, and maximum response latencies by listener gender and vocalizer gender; $X$ represents group means.

Duration Modified stimuli were omitted for this graph. Asterisk indicates significance, $p<.05$, with the following numbers indicating the estimate of the true difference of the means and the $95 \%$ confidence intervals.

* Female voc. - Male voc.:

$-136.84[-45.35,-228.32]$

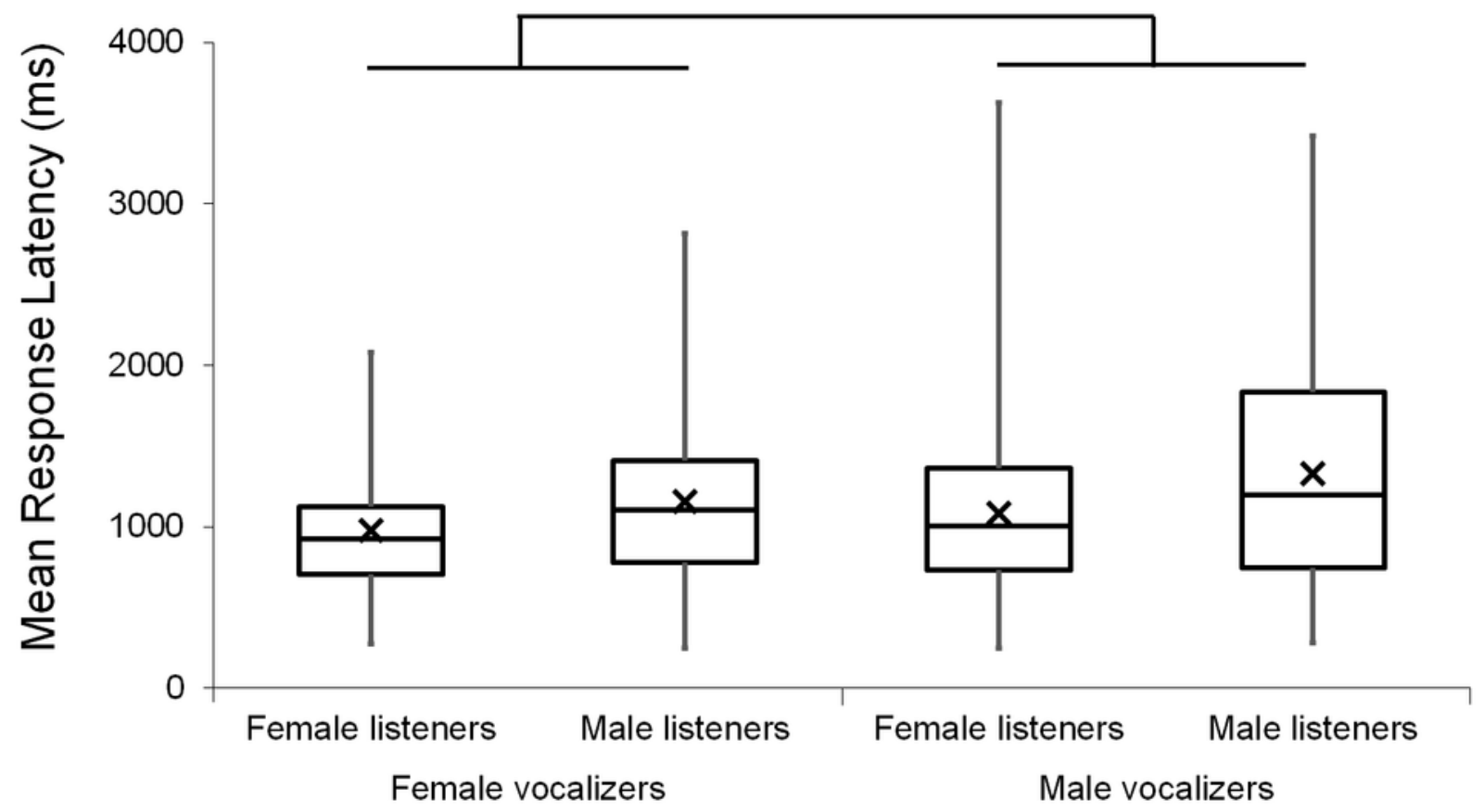

